\title{
Name Data Networking for Interplanetary Internet: An Architectural Perspective
}

\author{
Ritika Kumari ${ }^{1}$, R. L. Ujjwal ${ }^{2}$ \\ USICT Guru Gobind Singh Indraprastha University ${ }^{1}$, USICT Guru Gobind Singh Indraprastha University ${ }^{2}$ \\ Email: ritikakumari.ggsipu@gmail.com ${ }^{1}$,ujjwal@ipu.ac.in ${ }^{2}$
}

\begin{abstract}
There are several challenges when communicating in space. We do not have fixed infrastructure and have long delays, the probability of high error, intermittent connectivity and disruption of the link. These challenges draw the attention for improving communication challenges in spaces technologies and networking architectures. The TCP/IP stack is not effective in handling challenging environments and has issues of compatibility with the present security protocols. An alternate solution is Delay Tolerant Networking Architectures. It specifies a bundle protocol. This protocol resides on the top of the transport layer. The Delay Tolerant Networking architecture provides information storage for a longer duration on transitional nodes. It is appropriate when encountering disrupted links, long links, and intermittent connectivity. NDN focuses on content instead of IP addresses making it a reliable and robust architecture for the future Internet. In NDN, there is no need for setting up and maintenance of steady paths between end nodes. It makes NDN suitable for network with intermittent connectivity and very large propagation delays. This paper discusses the perspective of integrating Delay Tolerant Networking with Name Data Networking.
\end{abstract}

Keywords - Name Data Networking, Interplanetary Internet, Delay Tolerant Networking.

\section{INTRODUCTION}

Interplanetary Internet defines the architecture \& protocols that are important for permitting interoperation of the Internet systems on Earth/Spacecraft [6]. It involves communication between two entities when both of them are present in the space or when one entity is present on the ground and the other entity is there in space. With the evergrowing development in space invocations, empowering the acknowledgment of future space investigations missions, Interplanetary Networks are the next step ahead that will result in efficient and robust communication infrastructure. In such unfavorable and uncertain environment, our traditional protocols like TCP/IP do not perform as required. Therefore, there is a need to have a new architecture that can support interplanetary communication efficiently. There are several issues that come into the picture when we deal with Interplanetary Internet. We have extremely large and variable propagation delays in space. For maintaining a communications link between two spacecraft, we have two links-one forward physical and one or more multiple returns physical links. The challenge is that these links have asymmetrical capacities. It is impossible to establish and maintain a path, so there is a lack of fixed communication infrastructure. There is also a backward compatibility requirement because high investment is involved. There are other challenges like power, mass, size, and cost.

Name Data Networking is one among the projects funded by the U.S. National Science Foundation under its FIA [2]. The roots of name data networking are in content centric networks [12]. In NDN, data packets with content names are used. These names are unique allowing the network routers to monitor and keep track of packet states. Security in NDN is ensured as producer signs all the data packets and verification of the data packets is done by the consumers. Multi-path forwarding is also supported by the routers in
Name Data Network (NDN). The paper organization is as follows. Section II discusses the overview of name data networking, its classification, architecture, and working principle. A brief description of Interplanetary Networks and its architecture is given in section III. In section IV, we discuss how NDN is based on Interplanetary Networks/communication. Finally, conclusions are drawn.

\section{NAME DATA NeTWORKING}

In NDN, data packets with content names are used. The data packets are independent and self-contained. NDN has an hour-glass architecture. If we talk about the OSI model, there is IP working in the network layer [11]. There is a problem if we want to introduce new functionalities to the IP. Existing protocols are not suitable for accommodating nay change. In order to be a future Internet architecture, network layer of NDNs must provide solution for four major issues security, scalability, resiliency, and efficiency [7]. We have two more layers present in NDN protocol stack: security and strategy. The function of security layer is to provide security to every content present in packets. The Strategy layer deals with forwarding decision for all requests of incoming content. Transport layer as we know it is not present in NDN [14]. The services provided by the transport layer are embedded into the NDN forwarding plane [9]. 


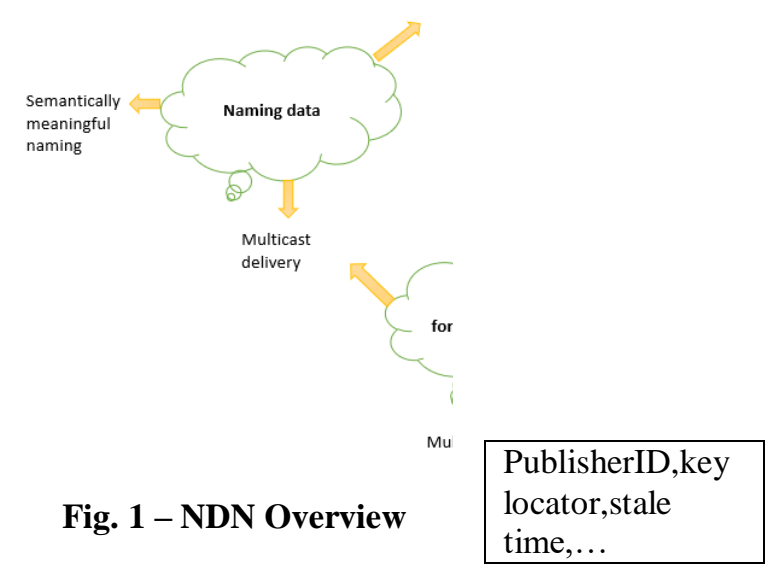

A. NDN Classification

We have classified Name Data Networking features as NDN applications, system services and system architecture [11].

- Applications: It deals with the real-life benefits of NDN

- System Services: Main Functions of NDN lies here, i.e. mobility, privacy and trust, routing, storeand-forward, security, etc.

- System Architecture: Here lies the Component organization as well as interaction mechanism between them

\section{B. Architecture and Working Principles of NDN System}

In Name Data Network (NDN), communication is started with the help of an I-pkt i.e. interest packet by the consumer [13]shown in Fig. 2(a). A Data Packet D-packet will be issued for the I-packet only when the I-packet shown in Fig. 2(b) [1].

reaches a node having the valid content. Content Names are embedded in both packets. D-pkt will travel back to the consumer by retracing the route of the interest packet. Each Router in NDN works by maintaining in 3 Data Structures [1].

- Content Store: A copy of D-pkt is kept by each router in the content store till the time they are replaced by some new data.

- Pending Interest Table (PIT): For every I-pkt, there will be an entry in PIT. This entry will be stay as long as its corresponding D-pkt arrives or the entry's lifetime expires, whatever happens earlier.

- Forwarding Information Base (FIB): Its job is to maintain the next-hop(s) and related information for every destination name prefix which is reachable.

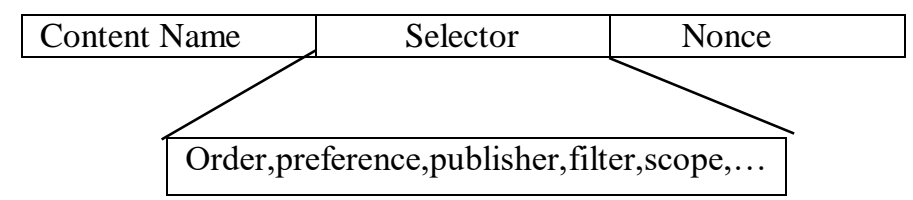

(b) D_pkt

\begin{tabular}{|c|c|c|c|}
\hline $\begin{array}{l}\text { Content } \\
\text { Name }\end{array}$ & Signature & Signed info & Data \\
\hline $\begin{array}{l}\text { Digest } \\
\text { witness, }\end{array}$ & algorithm, & & \\
\hline
\end{tabular}

Fig. 2 - Packets in NDN Architecture
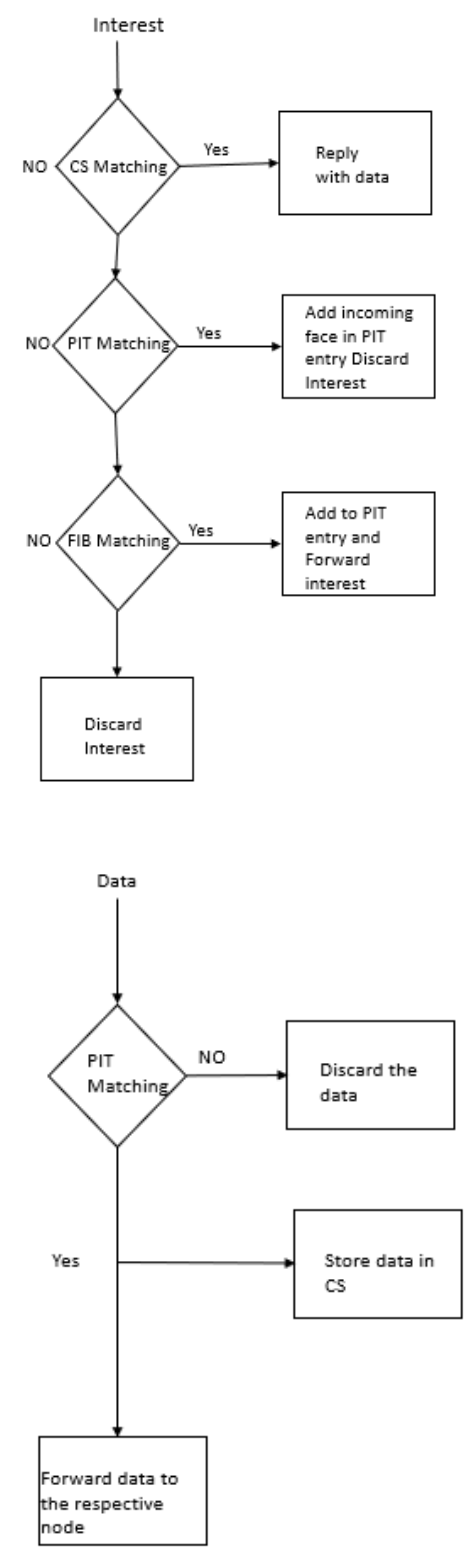

Fig. 3 - Flow diagram of packets in NDN 


\section{INTERPLANETARY NETWORKS: HISTORY AND ARCHITECTURE}

\section{A. Challenges}

There are various challenges that we come across when dealing with the Interplanetary Networks. These are: extremely variable and large propagation delays; the presence of asymmetrical Up and Down link capacities; the potentially higher error; insufficient fixed communication infrastructure; power, mass and investment in hardware; requirement of backward compatibility. These issues undermine the efficiency and reliability of the communications process [3]. These challenges encourage further developments and enhancements of space communications technologies.

\section{B. Interplanetary Networks Architecture}

Interplanetary Networks mainly comprises of 3 networks:

- IPN Backbone Network: As there is a need for huge capacity and always available link, IPN Backbone Network provides these services.

- IPN External Networks: This consists of nodes having short and long-distance communication capabilities.

- PN Networks: It comprises of Satellite and surface Network on Planet. Further the

The PN Surface network helps in communication links between surface elements, such as rovers, landers, and sensors on the surface of planets whereas PN Satellite Network provide the communication between the satellites.

\section{DTN BASED ON INTERPLANETARY NETWORKING/COMMUNICATION}

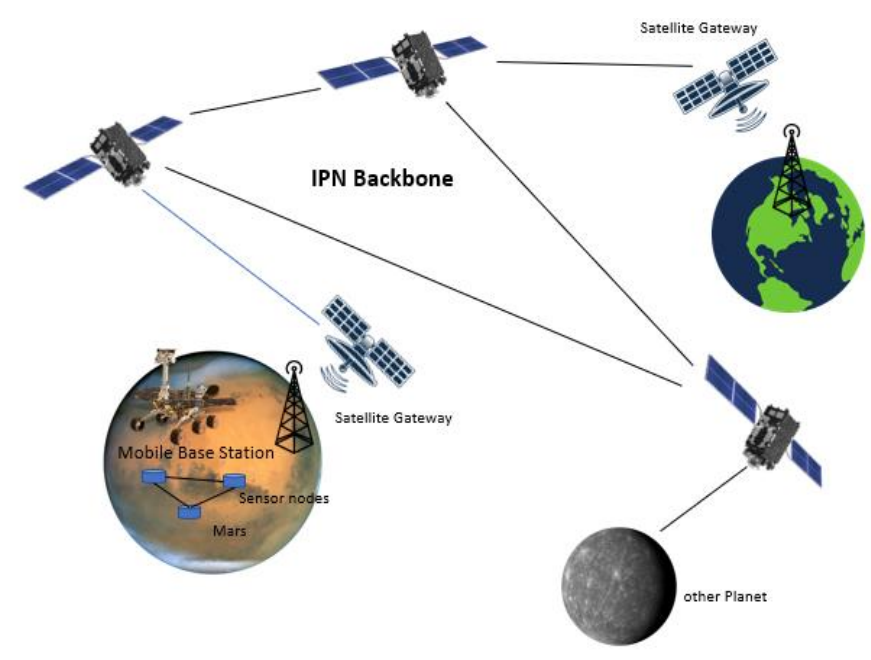

Fig. 4 - Interplanetary Communication

Ongoing improvements in space interchanges have begun tending to current difficulties. Consultative Committee for
Space Data Systems (CCSDS) has built up different norms for space interchanges. CCSDS File Delivery Protocol (CFDP) is designed for reliable IPN file transfer.

\section{A. PROTOCOL STACK}

Both CCSDS protocol and the OSI Model Protocol Architecture are close to each other. But the protocols working in each layer of CCSDS are way more complex. They can support the unfavorable environment of space [6].

There are 8 layers working:

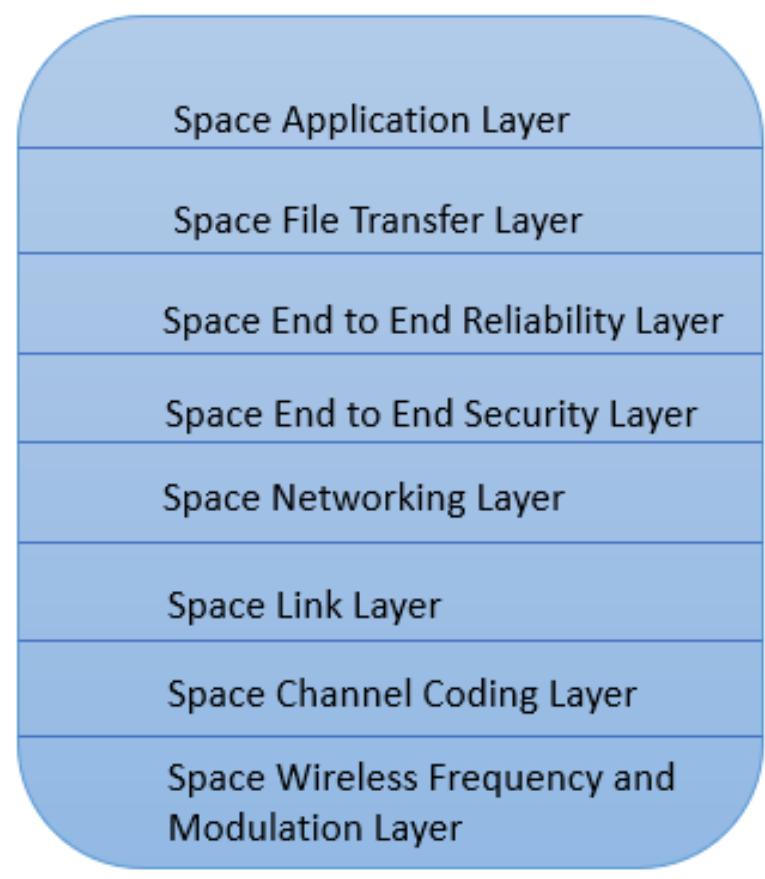

Fig. 5 - CCSDS Protocol Stack

1. Space Application Layer: End to End application services like transferring files, compression of data etc. are provided by the protocols in this layer.

2. Space file transfer layer: This layer provide support for two protocols: (1) FTP (File Transfer Protocol); (2) CCSDC File Delivery Protocol.

3. Space End to End Reliability Layer: Reliability from End to End is ensured by this layer.

4. Space End to End security Layer: In this layer, multiple levels of data protection is provided by IPSec and SCPS security protocol.

5. Space Networking Layer: This layer supports static as well as dynamic routing mechanisms.

6. Space link layer: It contains the methods of passing data units by the upper layers over the link. 
7. Space Channel Coding Layer: Two important roles are: synchronization and channel coding over space.

8. Space Wireless Frequency and Modulation Layer: Frequencies and efficient types of modulations for creating the channel connecting the two spacecraft are specified.

\section{B. Brief of CFDP}

CFDP is an approach for reliable transmission that can bear comparably variable and long round-trip latency [4]. There are two modes of CFDP operation. The modes are unacknowledged and acknowledged mode.

In the latter, if the data is corrupted or lost then it will be retransmitted automatically.

\section{ARCHITECTURE OF CFDP}

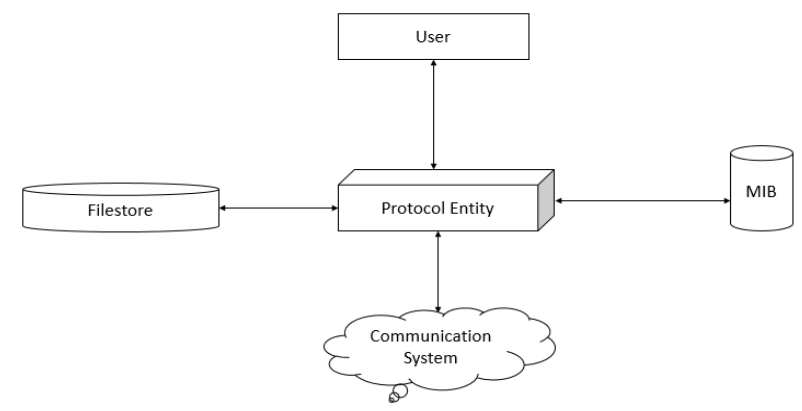

Fig. 6 - Architectural elements in CFDP

\section{Elements of CFDP Protocol}

1. Protocol Entity: Core delivery procedures are implanted by the protocol entity.

2. User: User must be present for the protocol to operate.

3. File Store: It is the storage service provided by the CFDP.

4. Communication System: It is also conveyed as a Unit Data Transfer layer where all the entities have access.

5. Management Information Base (MIB): As the data is static in nature usually, it can be stored in MIB as System Tables.

We have discussed the challenges that we face in space communications. The TCP/IP stack cannot handle this kind of challenging environments [6]. Delay Tolerant Networking architecture provides an alternative solution by specifying an overlay protocol known as Bundle protocol.

\section{DTN Architecture and Bundle protocol}

We have an overlay on top of transport layer or other layer protocols. The significance of overlays is that at each DTN hop, delays and disruptions can be tackled [5]. In DTN architecture, application data is stored on intermediate nodes before it is being forwarded to the next node on the path.

The Bundle Protocol is the most widely used DTN protocol. The elementary unit of data is" Bundle" in this protocol. Bundle consists of destination names, sender, data units and and other information that is necessary for an end to end communication [5]. Using convergence layer adapters (CLA), BP can be interfaced with different lower layer protocol networks. We can summarize DTN as:

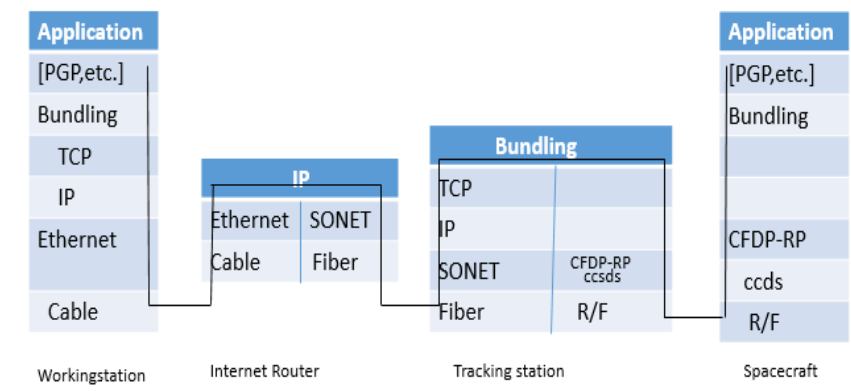

Fig. 7 - Bundling based Interplanetary Internet.

\section{- DTN as an overlay}

As explained earlier, we have an overlay on top of Transport layer or other lower layer protocols. The end to end path maybe disintegrated into multiple DTN hops [5] simply by installing a bundle protocol agent on the endpoints and nodes at the border of homogeneous segments.

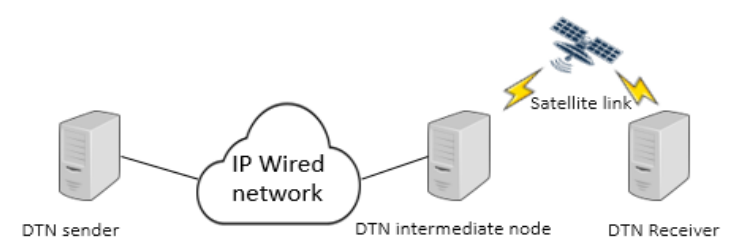

Fig. 10 - DTN as an Overlay

\section{- Information Storage at Intermediate Nodes}

On the basis of information storage, we can say that there is stark difference between TCP/IP and DTN networks. In networks where we have better connectivity and minuscular delays, routers rely on short-term storage and information is persistently cached only at end nodes [15]. The information can be accessed directly from the source as the transmission is reliable.

Obviously, it isn't the scenario with IPN. To deal with these challenges, information is stored for a longer duration at intermediate DTN.

\section{E. DTN without Bundling}


Here, we see that without any modifications to the protocol, the development of supporting infrastructure will enable UDP/IP to work more like bundling [4] and will enable the deployment of DTN to be built on present capabilities. As IP protocol mediates with different link layer protocols i.e., we have a reliable system which uses TCP/IP tunnels and afterward, we built IP virtual interfaces to this RLT system, CFDP-RP and also to other systems. This provides end-toend reliability to IP over heterogeneous links. Without performing protocol modifications, it becomes a challenge to have the Bundling capability within the Internet model.

The strategy is as follows:

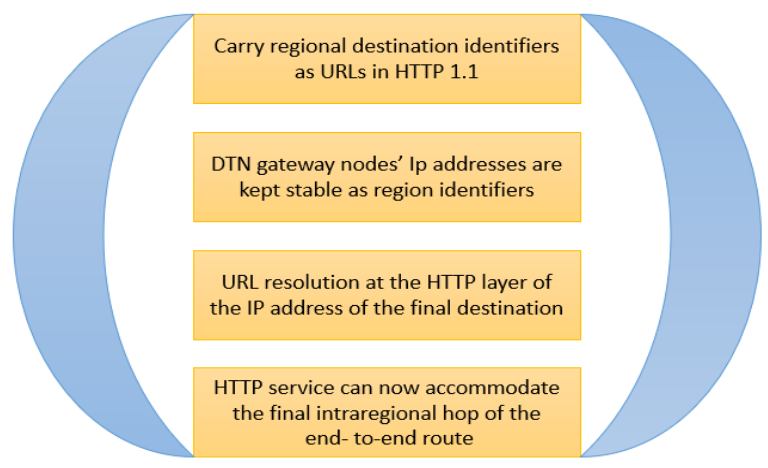

Fig. 8 - Integration of DTN and NDN Strategy

\section{NDN BASED INTERPLANETARY NETWORKING/COMMUNICATION}

Name Data Networking was not developed for high-delay networks rather it was meant to be for low delay and highly connected networks environments. There is inefficiency in the case where we have intermittent connectivity. So, there is a need for some additional mechanisms. The challenge that we consider in this paper is to integrate NDN and DTN in order to provide support for delay intermittent connectivity. First, try to build delay/disruption tolerant functionality into NDN i.e., Data is lost when transferred back, as a result, the consumer has to send interest packet again, but is not efficient. Second, by integrating DTN, one can use the existing implementations rather than implementing delay/disruption-tolerant functionality from the scratch in NDN. It is like viewing DTN as an NDN underlay.

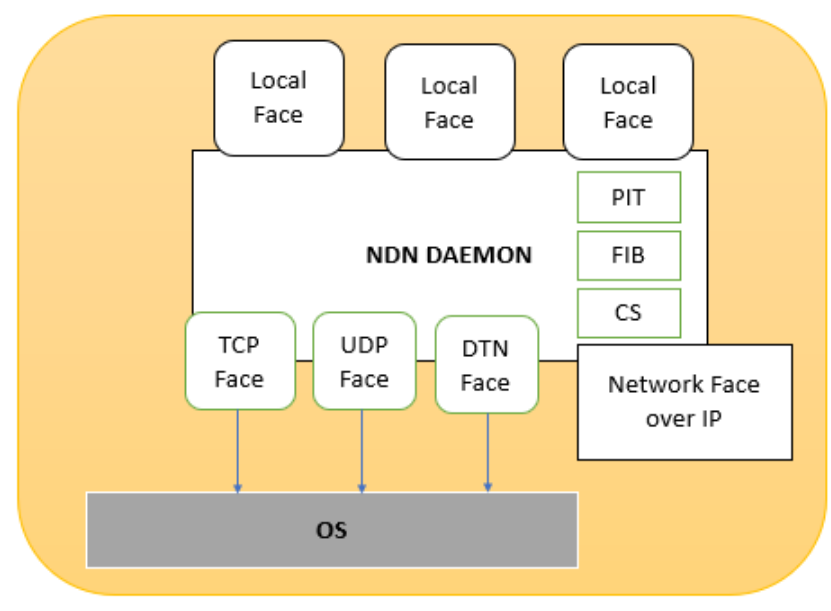

Fig. 9 - NDN-DTN Based Interplanetary Internet architecture.

The DTN architecture is suitable for 3 scenarios [5]:

- Geostationary Earth Orbit Satellites with fixed terminals

- Geostationary Earth Orbit with mobile terminals

- Lower Earth Orbit Satellites

Geostationary Earth Orbit Satellites with fixed terminals: With fixed terminals, end-to-end connectivity is mostly viable.

Geostationary Earth Orbit with mobile terminals: DTN can potentially increase resilience here.

Lower Earth Orbit Satellites: As no End to end connectivity is present here DTN can be of great utility here.

\begin{tabular}{|l|l|}
\hline \multicolumn{1}{|c|}{ Pros } & \multicolumn{1}{|c|}{ Cons } \\
\hline $\begin{array}{l}\text { DTN are compatible with the NDN } \\
\text { architecture. This approach of integration } \\
\text { between NDN and DTN is allows the ND } \\
\text { deployment over the existing DTN } \\
\text { implementations. }\end{array}$ & $\begin{array}{l}\text { NDN is not a native mechanism and it is } \\
\text { not a delay tolerant by itself. }\end{array}$ \\
\hline $\begin{array}{l}\text { Abstraction of intermittent connectivity } \\
\text { from NDN }\end{array}$ & $\begin{array}{l}\text { Overhead is there due to the presence of } \\
\text { additional layers. }\end{array}$ \\
\hline
\end{tabular}

Fig. 10 - Approach Tradeoffs

\section{CONCLUSION}

This paper presented a brief description of Name Data Networking and its classification. It also provided the architecture and the working principle. We discussed the interplanetary networks and the architecture. A brief of DTN based interplanetary networking/communication, protocol stack, CFDP protocol, and its elements are presented. We discussed DTN architecture and bundle protocol and an overview of DTN without bundling. Finally, we mentioned NDN based interplanetary networking/communication. Moreover, we give an insight into how Interplanetary Network can be based on Name Data Networking. 


\section{REFERENCES}

[1] Saxena. D.. Ravchoudhurv. V.. Suri. N.. Becker. C. and Cao. J.. 2016. Named data networking: a survey. Computer Science Review, 19, pp.15-55.

[2] Pan. I.. Paul. S. and Jain. R.. 2011. A survev of the research on future internet architectures. IEEE Communications Magazine, 49(7), pp.26-36.

[3] Wu. H.. Li. Y. and Cao. B.. 2015. November. Internlanetarv communication technologies. architectures and annlications. In 2015 IEEE/CIC International Conference on Communications in China (ICCC) (pp. 1-6). IEEE.

[4] Burleigh. S.. Hooke. A.. Torgerson. L.. Fall. K.. Cerf. V.. Durst. B.. Scott. K. and Weiss. H.. 2003. Delavtolerant networking: an annroach to internlanetarv internet IFEE Communications Magazine, 41(6), pp.128-136.

[5] Caini. C.. Cruickshank. H.. Farrell. S. and Marchese. M.. 2011. Delav-and disruntion-tolerant networking (DTN): an alternative solution for future satellite networking annlications. Proceedings of the IEEE, 99(11), pp.1980-1997.

[6] Rahman. K.S.. Islam. M.M. and Kabir. M.H.. 2014. March. Internlanetarv network: A brief introduction. In 16th Int'l Conf. Comnuter and Information Technology (pp. 261-266). IEEE.

[7] Ding. W.. Yan. Z. and Deng. R.H.. 2016. A survev on future Internet security architectures. IEEE Access, 4, pp.4374-4393.

[8] Potdar. V.. Sharif. A. and Chang. E.. 2009. Wireless multimedia sensor networks: a survev. In Proceedings of the international workshon on securitv in RFID and its industrial annlications with IEEE 23rd international conference on advanced information networking and annlications. (AINA 2009) (pp. 636-641). IEEE Computer Society.

[9] Yi C. Afanasvev A. Moiseenko I. Wang L. Zhang B. Thang I. A case for stateful forwarding nlane. Computer Communications. 2013 Apr 1;36(7):779-91.

[10]Baccelli E. Mehlis C. Hahm O. Schmidt TC. Wählisch M. Information centric networking in the IoT: Exneriments with NDN in the wild. InProceedings of the $1 \mathrm{st}$ ACM Conference on Information-Centric Networking 2014 Sep 24 (pp. 77-86). ACM.

[11]Burke J. Gasti P. Nathan N. Tsudik G. Securing instrumented environments over content-centric networking: the case of lighting control and NDN. In2013 IEEE Conference on Comnuter Communications Workshons (INFOCOM WKSHPS) 2013 Apr 14 (pp. 394-398). IEEE.

[12]Xvlomenos G. Ververidis CN. Siris VA. Fotiou N. Tsilonoulos C. Vasilakos X. Katsaros KV. Polvzos GC. A survev of information-centric networking research. IEEE Communications Surveys \& Tutorials. 2014 May;16(2):1024-49.

[13]Perino D. Varvello M. A realitv check for content centric networking. InProceedings of the ACM SICCOMM workshon on Information-centric networking 2011 Aug 19 (pp. 44-49). ACM.

[14] Yuan H. Song T. Crowlev P. Scalable NDN forwarding: Concents. issues and princinles. In2012 $21 \mathrm{st}$ International Conference on comnuter communications and networks (ICCCN) 2012 Jul 30 (pp. 1-9). IEEE.

[15]Balasubramanian A. Levine B. Venkataramani A. DTN routing as a resource allocation problem. InACM SICCOMM Comnuter Communication Review 2007 Aug 27 (Vol. 37, No. 4, pp. 373-384). ACM. 